\title{
Expression of HGF and c-Met Proteins in Human Keratoconus Corneas
}

\author{
Jingjing You, ${ }^{1}$ Li Wen, ${ }^{1}$ Athena Roufas, ${ }^{1}$ Chris Hodge, ${ }^{1,2}$ \\ Gerard Sutton, ${ }^{1,2,3}$ and Michele C. Madigan ${ }^{1,4}$ \\ ${ }^{1}$ Save Sight Institute, Discipline of Clinical Ophthalmology, The University of Sydney, Sydney, NSW 2000, Australia \\ ${ }^{2}$ Vision Eye Institute, Chatswood, NSW 2067, Australia \\ ${ }^{3}$ Auckland University, Auckland 1010, New Zealand \\ ${ }^{4}$ School of Optometry \& Vision Science, UNSW, Kensington, NSW 2052, Australia
}

Correspondence should be addressed to Jingjing You; jing.you@sydney.edu.au

Received 7 July 2015; Revised 30 October 2015; Accepted 12 November 2015

Academic Editor: Neil Lagali

Copyright (c) 2015 Jingjing You et al. This is an open access article distributed under the Creative Commons Attribution License, which permits unrestricted use, distribution, and reproduction in any medium, provided the original work is properly cited.

Keratoconus $(\mathrm{KC})$ is a progressive degenerative inflammatory-related disease of the human cornea leading to decreased visual function. The pathogenesis of $\mathrm{KC}$ remains to be understood. Recent genetic studies indicate that gene variants of an inflammationrelated molecule, hepatocyte growth factor $(H G F)$, are associated with an increased susceptibility for developing KC. However HGF protein expression in KC has not been explored. In this initial study, we investigated late-stage KC and control corneas for the expression of HGF and its receptor mesenchymal-epithelial transition factor (c-Met/Met). KC buttons ( $\sim 8$ mm diameter) $(n=10)$ and whole control corneas $(n=6)$ were fixed in $10 \%$ formalin or $2 \%$ paraformaldehyde, paraffin embedded and sectioned. Sections were immunolabelled with HGF and c-Met antibodies, visualised using immunofluorescence, and examined with scanning laser confocal microscopy. Semiquantitative grading was used to compare HGF and c-Met immunostaining in KC and control corneas. Overall, KC corneas showed increased HGF and c-Met immunostaining compared to controls. KC corneal epithelium displayed heterogeneous moderate-to-strong immunoreactivity for HGF and c-Met, particularly in the basal epithelium adjacent to the cone area. Taken together with the recent genetic studies, our results further support a possible role for HGF/c-Met in the pathogenesis of KC.

\section{Introduction}

Keratoconus (KC) is the most common primary human degenerative corneal disease with a prevalence of around 1 in 2000 worldwide [1]. It is bilateral, asymmetric, and progressive, leading to corneal thinning and irregularity [2]. Onset primarily occurs in the 2nd decade of life and is associated with significant decreasing visual function [2] and morbidity [3]. $\mathrm{KC}$ is the main indication recorded for corneal grafts in Australia [4], and currently its progression can only be halted through surgical interventions including collagen crosslinking that stiffens the cornea using riboflavin and UVA [5]. More recently a surgical procedure was developed transplanting isolated Bowman's layer from donor corneas to KC eyes as a further late-stage intervention [6].
The histopathology of $\mathrm{KC}$ is well described and includes epithelial and stromal thinning within the apical cone region, breaks in the Bowman's layer, focal fibrosis, and anterior stromal keratocyte apoptosis $[2,7]$. However the underlying pathogenesis of $\mathrm{KC}$ remains unclear. Recent evidence indicates a role for inflammation in the disease, with increased recruitment of inflammatory cells (e.g., macrophages, lymphocytes, and antigen presenting cells) [8] and inflammatory markers such as interleukin-1 (IL-1) and transforming growth factor-beta (TGF- $\beta$ ) [9] observed in KC corneal tissue sections. Increased expression of inflammatory markers such as interleukin-6 (IL-6), tumour necrosis factor alpha (TNF- $\alpha$ ), and matrix metalloproteinase 9 (MMP-9) has also been found in tears collected from KC patients compared to controls [10]. Furthermore, a recent review examining the biochemical 
TABLE 1: Characteristics of KC patients.

\begin{tabular}{|c|c|c|c|c|c|c|}
\hline${ }^{\mathrm{a}} \mathrm{KC}$ & Gender & Age at diagnosis (yrs.) & Age at surgery (yrs.) & Contact lenses ( $\mathrm{Y}$ or $\mathrm{N})$ & $\begin{array}{l}\text { History of allergy and/or atopy } \\
\text { (Y or N) }\end{array}$ & ${ }^{\mathrm{b}} \mathrm{DALK}(\mathrm{Y}$ or $\mathrm{N})$ \\
\hline 1 & $\mathrm{~F}$ & 24 & 32 & $\mathrm{Y}$ & $\mathrm{N}$ & $\mathrm{N}$ \\
\hline 2 & $\mathrm{~F}$ & 23 & 30 & $\mathrm{~N}$ & $\mathrm{Y}$ asthma & $\mathrm{Y}$ \\
\hline 3 & $\mathrm{~F}$ & 30 & 37 & $\mathrm{~N}$ & $\mathrm{~N}$ & $\mathrm{Y}$ \\
\hline 4 & $\mathrm{M}$ & 27 & 32 & $\mathrm{~N}$ & $\mathrm{Y}$ atopy and asthma & $\mathrm{N}$ \\
\hline 5 & $\mathrm{M}$ & 25 & 32 & $\mathrm{~N}$ & $\mathrm{~N}$ & $\mathrm{Y}$ \\
\hline 6 & $\mathrm{~F}$ & 18 & 21 & $\mathrm{~N}$ & $\mathrm{Y}$ atopy and asthma & $\mathrm{N}$ \\
\hline 7 & M & 24 & 28 & $\mathrm{Y}$ & $\mathrm{N}$ & $\mathrm{N}$ \\
\hline 8 & M & 24 & 43 & $\mathrm{Y}$ & $\mathrm{N}$ & $\mathrm{N}$ \\
\hline 9 & $\mathrm{~F}$ & 20 & 31 & $\mathrm{Y}$ & $\mathrm{N}$ & $\mathrm{N}$ \\
\hline 10 & $\mathrm{~F}$ & 32 & 38 & $\mathrm{Y}$ & $\mathrm{N}$ & $\mathrm{Y}$ \\
\hline
\end{tabular}

${ }^{\mathrm{a}}$ Grade 4 KC: severe; VA >6/7.5 with contact lens correction; severe corneal thinning and Munson's sign.

${ }^{\mathrm{b}}$ DALK: deep anterior lamellar keratoplasty.

changes in KC proposed a "two-hit hypothesis" with a "genetic predisposition to the corneal disease and a second hit that may induce abnormalities of inflammatory components" [11].

Single nucleotide polymorphism (SNP) refers to a change in a single nucleotide within a DNA sequence and is the most common type of genetic variation observed in the human genome [12]. SNPs have been widely studied as genetic markers for human disease. Two parallel genome-wide association studies identifying potential SNPs associated with KC, using independent sample cohorts, reported a significant association between $\mathrm{KC}$ and the hepatocyte growth factor (HGF) gene, identifying two single nucleotide polymorphisms (SNPs; rs3735520 and rs17501108) in the promoter region [13]. Further, Burdon et al. (2011) also examined HGF protein abundance in the serum of controls correlating to the rs3735520 genotype and found a significant increase in HGF serum protein associated with the minor allele $\mathrm{T}$ [13].

HGF is a pleiotropic growth factor that activates the $\mathrm{HGF} / \mathrm{c}-\mathrm{Met}$ pathway after binding to its receptor, mesenchymal-epithelial transition factor (c-Met/Met). Once activated, downstream pathways such as mitogen-activated protein kinase (MAPK) cascades, PI3K-Akt axis or Janus kinase/signal transducers, and activators of transcription (JAK/STAT) pathways may be activated [14]. HGF has been implicated in several cellular roles within the cornea. For example, together with MMP-1, HGF is reported to initiate human corneal epithelial cell migration in vitro [15], and exogenous HGF has been found to promote the proliferation of both corneal epithelial and endothelial cells [16]. In injured rabbit corneas, Wilson et al. (1999) reported an obvious increase of HGF mRNA expression in keratocytes and $c$-Met mRNA expression in epithelial cells compared to unwounded corneas, suggesting that the HGF/c-Met pathway plays a role in corneal wound healing [17]. Studying bovine corneal wound healing in organ culture models, Carrington and Boulton (2005) showed that HGF delayed epithelial layer formation, together with increased differentiation of keratocytes to myofibroblasts, compared with untreated and keratinocyte growth factor (KGF) treated corneas [18].

The expression of HGF and c-Met proteins in human $\mathrm{KC}$ corneas has not been investigated to date. One study has reported increased serum HGF expression for at least the minor allele of HGF SNP rs3735520, associated with increased potential for developing KC [13]. As a first step in assessing the role of HGF protein and its receptor (c-Met) in $\mathrm{KC}$, we used corneal buttons from patients with severe $\mathrm{KC}$ and control human corneas to compare and examine the distribution and expression of these proteins.

\section{Materials and Methods}

KC corneal tissue buttons (Vision Eye Institute, Chatswood, NSW Australia) and donor corneas (Lions New South Wales (NSW) Eye Bank) were obtained with consent and approval from the Sydney Eye Hospital Human Research Ethics Committee (HREC 2013/1041). All procedures were in accordance with the Declaration of Helsinki. Informed consent was obtained from all participants prior to collection of KC buttons. Normal donor corneas were obtained from the Lions NSW Eye Bank following appropriate consent and HREC approval.

2.1. Corneal Specimens. Ten corneal buttons were collected from KC patients (age range from 18 to 32 years) undergoing corneal transplantation at Vision Eye Institute. All KC patients were diagnosed on the basis of clinical signs and corneal topography and were classified as KC grade 4 (most severe stage) (Table 1). Six normal donor corneas (age range 53 to 83 years) were obtained from the Lions NSW Eye Bank (Table 2).

KC corneal buttons ( $~ 8 \mathrm{~mm}$ diameter), with the cone apex location marked, were fixed in $10 \%$ neutral buffered formalin (NBF). Whole corneas were fixed in $2 \%$ paraformaldehyde/PBS ( $\mathrm{pH} 7.4$ ). All specimens were paraffin embedded and cut at $6 \mu \mathrm{m}$. Sections were collected on Super-Frost Plus 


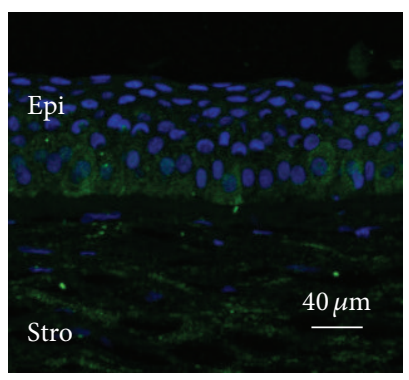

(a)

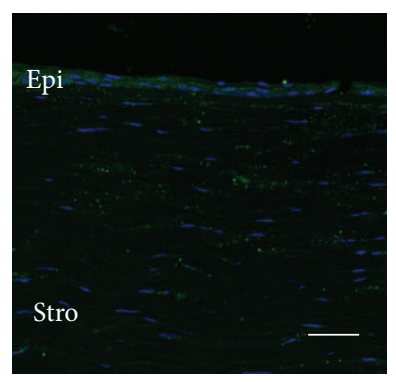

(b)

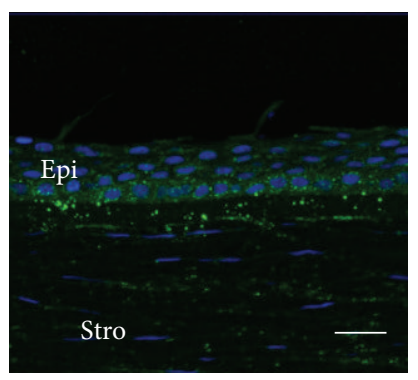

(c)

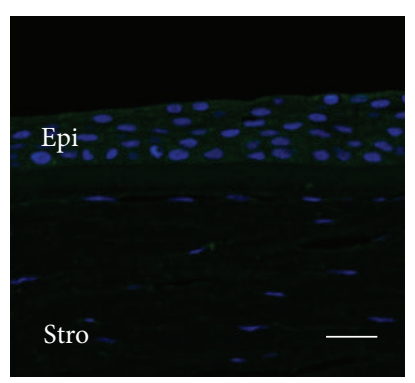

(d)

FIGURE 1: Representative images showing increased HGF expression in basal epithelial cells adjacent to the cone region in KC (a), compared to the relatively more uniform expression seen in all layers of control cornea epithelium at a similar location (c). Strong HGF stromal staining (keratocyte and extracellular matrix) is also observed in $\mathrm{KC}$ and control corneas (a to c). No immunostaining is apparent in the Ig negative control (d). (a) Region adjacent to the cone in KC cornea, (b) cone region in KC cornea, (c) central region of control cornea, and (d) Ig negative control cornea. Epi: epithelium, Stro: stromal. Green: HGF; blue: nuclei.

TABLE 2: Characteristics of control corneas.

\begin{tabular}{lcc}
\hline Control & Gender & Age (yrs.) \\
\hline 1 & M & 67 \\
2 & F & 53 \\
3 & F & 64 \\
4 & F & 67 \\
5 & M & 83 \\
6 & M & 63 \\
\hline
\end{tabular}

slides (Menzel-Glaser, Saarbruckener, Germany) and dried before use.

2.2. Immunohistochemistry. Sections were dewaxed and rehydrated through alcohols to water. For antigen retrieval, sections were incubated in $0.01 \mathrm{M}$ citrate buffer $(\mathrm{pH} 6)$ at $85^{\circ} \mathrm{C}$ for 10 minutes, cooled to $40^{\circ} \mathrm{C}$, and rinsed in Tris-buffered saline (TBS, pH 7.4) with $0.1 \%$ Tween-20 (TBST). Sections were incubated at room temperature (RT) in 5\% bovine serum albumin (BSA) in TBST for 30 minutes, followed by incubation overnight at $4^{\circ} \mathrm{C}$ in $\mathrm{HGF}$ or c-Met primary antibodies, or appropriate negative controls (Mouse or Rabbit IgG) (Table 3). After overnight incubation, sections were washed in TBST and incubated in either goat anti-mouse Alexa Fluor 488 or donkey anti-rabbit Alexa Fluor 488.

For co-immunolabelling experiments, a separate series of slides were prepared and HGF visualised with Alexa Fluor 488 and c-Met visualised with Alexa Fluor 594, combined with nuclear Hoechst stain (Table 3).

Immunolabelling was repeated at least twice per specimen for each antibody, and appropriate Ig controls were included for each experiment. All slides were mounted in $20 \%$ glycerol/PBS, coverslipped, sealed with nail varnish, and viewed using a Zeiss LSM700 scanning laser confocal microscope and image software (Zen 2011, Carl Zeiss MicroImaging $\mathrm{GHBH}$, Jena, Germany). Where more than one colour was to be detected, multichannel excitation bleed-through was minimized using fluorochromes with separated peak excitations. Emission bleed-through was minimized by multitracking, where signal crosstalk between neighbouring channels was corrected by performing a sequential image capture routine.

2.3. Semiquantitative Analysis for HGF and c-Met. Semiquantitative grading of single-immunolabelled sections was used to assess the intensity and distribution of HGF and c-Met immunoreactivity in the corneal epithelium, stroma, and endothelium. Grading for KC buttons was made in the region adjacent to the cone (Adj) and for control corneas in a similar central corneal region. Immunolabelling of the thinned cone area of KC buttons was examined in each specimen but was not graded for comparison with controls because of the obviously altered morphology (only 1-2 epithelial layers present). The grading scale used was based on the intensity of the immunofluorescence $(0=$ no staining, $0.5=$ very weak, $1=$ weak, 2 moderate, and $3=$ strong) and the percentage (\%) area immunolabelled $(0=0 \%, 1=1 \%$ to $10 \%, 2=11 \%$ to $50 \%$, and $3>50 \%$ ) as described previously [19]. A final grade of 0 to 6 (intensity $+\%$ area immunolabelled) was then given for each specimen and this data is used to generate frequency histograms for HGF and c-Met immunostaining in $\mathrm{KC}$ and control specimens, respectively.

\section{Results and Discussion}

All KC corneas showed a thickened epithelium adjacent to the more central cone region (Figures 1(a) and 2(a)), compared to similar regions in the control corneas (Figures 1(c) and 2(c)), as we previously reported [19]. Only one to two layers of flattened epithelium were observed within the cone region (Figures 1(b) and 2(b)). Weak and more evenly distributed cytoplasmic immunostaining for HGF and c-Met was detected in the epithelium of control corneas (Figures 1(c) and 2(c)). In KC corneas, moderate-to-strong cytoplasmic immunostaining was seen within the basal and wing cell epithelial layers adjacent to the cone region (green fluorescence, Figures 1(a) and 2(a)). Only weak-to-moderate immunostaining of $\mathrm{HGF}$ and c-Met was detected in the $\mathrm{KC}$ cone region (Figures $1(\mathrm{~b})$ and $2(\mathrm{~b})$ ).

Co-immunolabelling for HGF and c-Met showed that c-Met (red) was primarily localised in the epithelium of 
TABle 3: Primary antibodies and negative controls used for immunostaining.

\begin{tabular}{llc}
\hline Primary antibody & Company & $\begin{array}{c}\text { Final } \\
\text { concentration } \\
\text { used }\end{array}$ \\
\hline Mouse anti-human HGF & Santa Cruz Biotechnology, Inc. (Dallas, Texas, USA) & $2 \mu \mathrm{g} / \mathrm{mL}$ \\
Rabbit anti-human c-Met & Santa Cruz Biotechnology, Inc. & $0.4 \mu \mathrm{g} / \mathrm{mL}$ \\
Mouse anti-human IgG & Zymed Laboratories (Thermo Fisher Scientific, Waltham, Massachusetts, & $2 \mu \mathrm{g} / \mathrm{mL}$ \\
Rabbit anti-human IgG & USA) & $0.4 \mu \mathrm{g} / \mathrm{mL}$ \\
Alexa Fluor 488 goat anti-mouse IgG & Zymed Laboratories & $2 \mu \mathrm{g} / \mathrm{mL}$ \\
Alexa Fluor 488 donkey anti-rabbit IgG & Molecular Probes (Life Technologies, Carlsbad, California, USA) & $2 \mu \mathrm{g} / \mathrm{mL}$ \\
Alexa Fluor 594 donkey anti-rabbit IgG & Molecular Probes & $2 \mu \mathrm{g} / \mathrm{mL}$ \\
Hoechst & Molecular Probes & $1 \mu \mathrm{g} / \mathrm{mL}$ \\
\hline
\end{tabular}

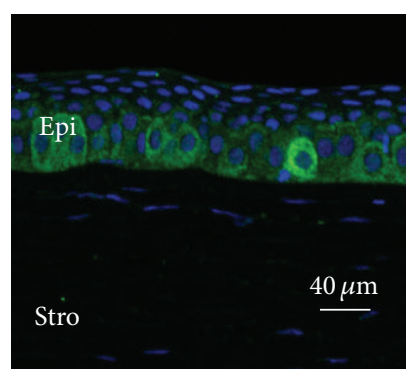

(a)

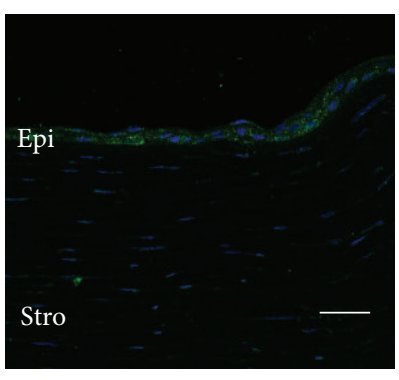

(b)

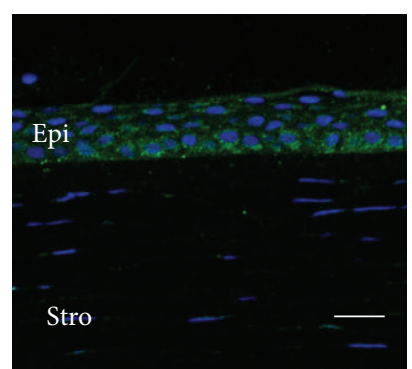

(c)

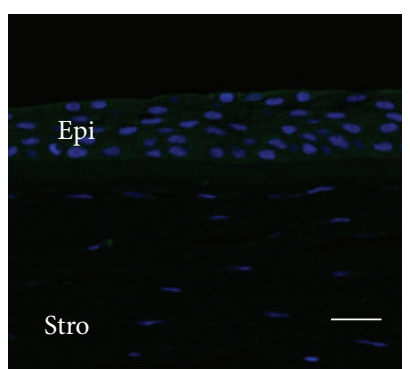

(d)

FIGURE 2: Representative images showing increased c-Met expression in basal epithelial cells adjacent to the cone region in KC (a), compared to the relatively more uniform expression seen in all layers of control cornea epithelium at a similar location (c). Weak c-Met staining is detected in the stroma in both KC and control corneas (a to c). No immunostaining is apparent in the Ig negative control (d). (a) Region adjacent to the cone in KC cornea, (b) cone region in KC cornea, (c) central region of control cornea, and (d) Ig negative control. Epi: epithelium; Stro: stromal. Green: HGF; blue: nuclei.

both control and KC corneas (Figure 3). HGF (green) was primarily localised within the stroma (both keratocytes and extracellular matrix) of control and $\mathrm{KC}$ corneas (Figure 3). Weak and relatively uniform immunostaining was observed in similar regions from control corneas for HGF and c-Met (Figure 3(a)). However, for KC corneas, increased HGF staining (green) was detected in the basal epithelium adjacent to the cone region and colocalised with increased c-Met staining (red) (Figure 3(d)). Limbal areas of control corneas showed slightly stronger stromal immunostaining of HGF compared to the central region of control corneas (Figure 3(b)).

Semiquantitative grading of HGF and c-Met immunostaining in $\mathrm{KC}$ samples was increased overall compared to the control corneas for similar regions, as indicated by the skewed distribution of the frequency histograms (Figures 4(a) and 4(b)). Overall, a greater proportion of KC corneas showed > grade 3 immunostaining for both HGF and c-met (KC: 70\% and $90 \%$, resp., versus control: $16 \%$ and $66 \%$ resp.) (Figures 4(a) and 4(b)).

Despite the importance of the HGF/c-Met pathway in regulating a number of key cellular activities, little is known about its potential role in normal or KC human corneas. As a first step to understand the possible role(s) of this pathway, we examined patterns of HGF and c-Met protein expression in both control and KC corneas. Higher levels of HGF and c-Met immunostaining were detected in the basal epithelium adjacent to the $\mathrm{KC}$ cone, compared to the weaker and more uniform epithelial staining pattern seen in control corneas.

In control corneas, we found HGF expression was more intense in the stroma compared to the epithelium. This is consistent with previous studies showing low level HGF mRNA expression in the human corneal epithelium compared to keratocytes and endothelium [16, 20]. The surface of normal rabbit corneal epithelium was reported to show only low level HGF protein [21]. Our immunostaining experiments detected stronger c-Met (HGF receptor) staining in epithelium compared to keratocytes in control corneas. This is consistent with $c$-Met mRNA findings, which showed that human corneal epithelium, keratocytes, and endothelium all expressed $c$-Met, but with highest mRNA expression in epithelium [16, 20,21]. These observations suggest that secreted keratocyte HGF may preferentially bind to the epithelium that expresses higher levels of c-Met (HGF receptor), compared to keratocytes that express low levels of c-Met, thus potentially regulating key epithelial cellular activities such as cell proliferation [22].

HGF is reported to be involved in two major processes, cell proliferation and migration, and inflammatory-related 

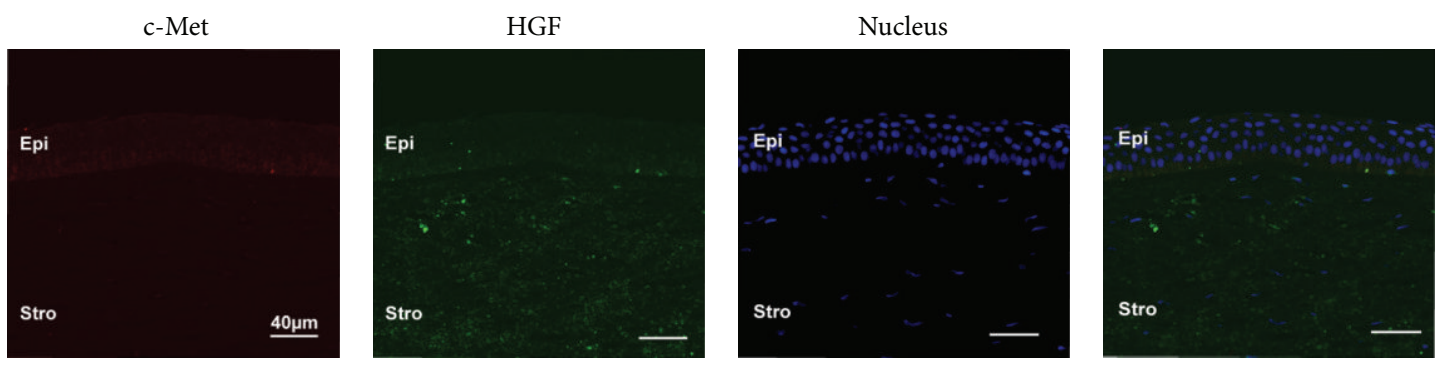

(a)
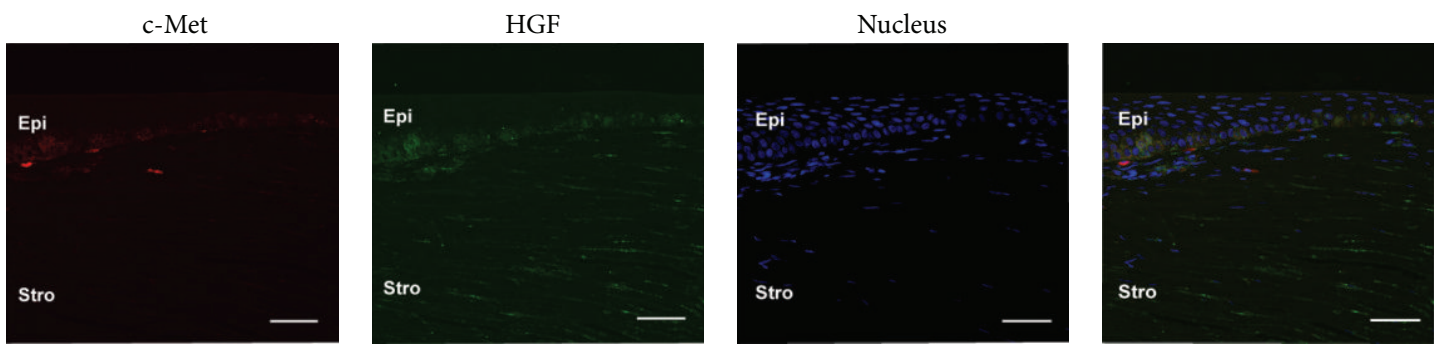

(b)
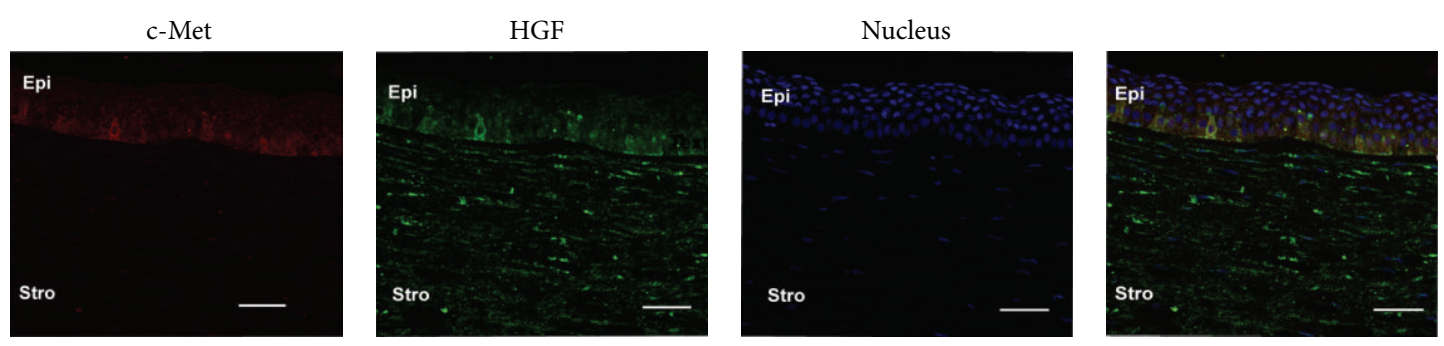

(c)
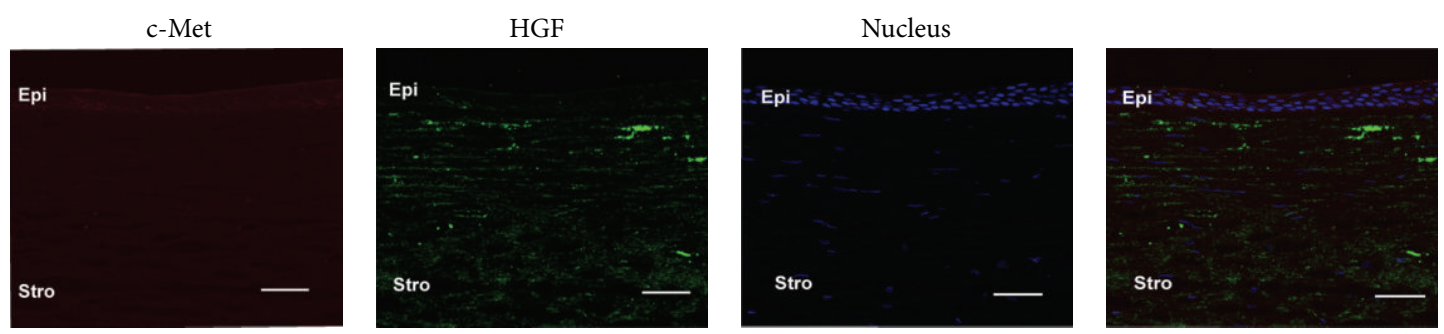

(d)
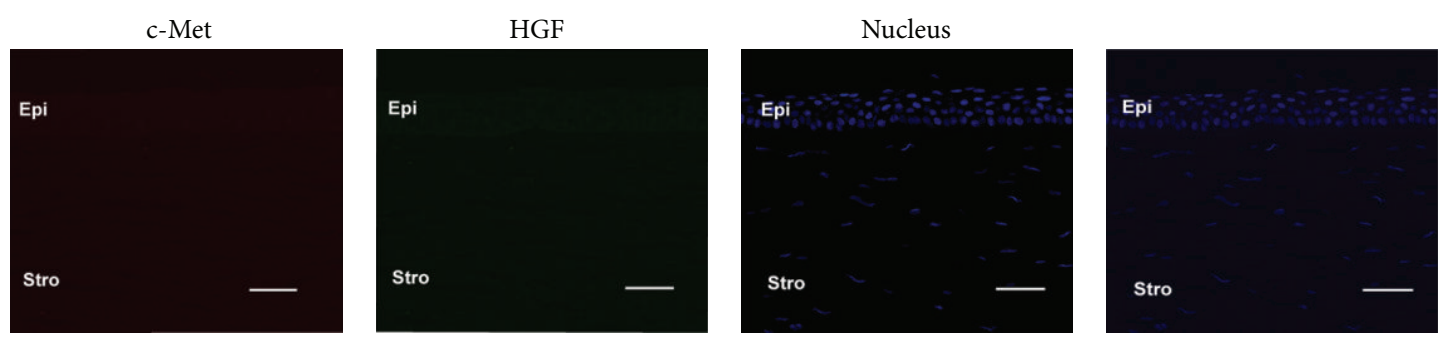

(e)

FIGURE 3: Representative images of central region of corneas with weak, uniform HGF (green), and c-Met (red) expression in all corneal layers (a). Patchy staining of HGF and c-Met is colocalised in the basal epithelium of both limbal regions (b) and adjacent to cone region of $\mathrm{KC}(\mathrm{c})$, with KC showing stronger staining than the limbal region. Strong HGF expression is seen within the stroma of both KC (c and d) and control corneas ( $\mathrm{a}$ and b). (a) Central region of control cornea, (b) limbal region of control cornea, (c) region adjacent to the cone in KC cornea, (d) cone region in KC cornea, and (e) Ms and $\mathrm{Rb}$ Ig control. 


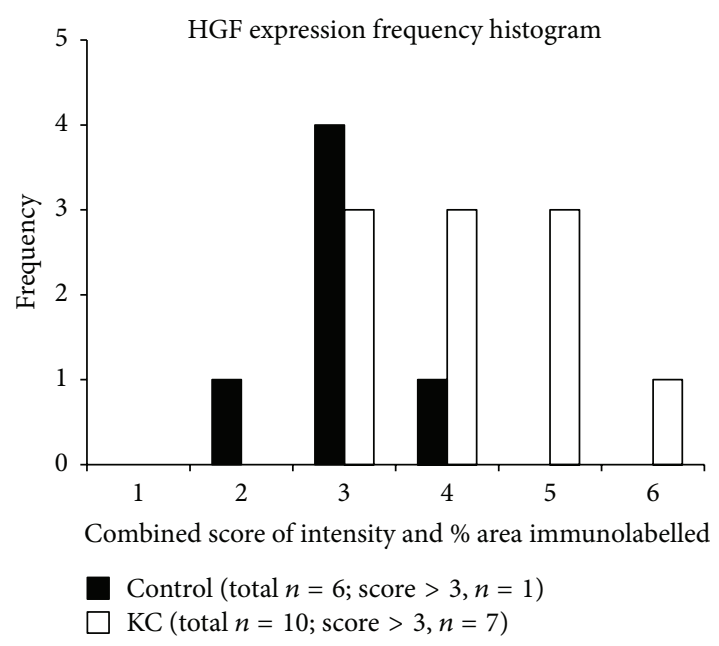

(a)

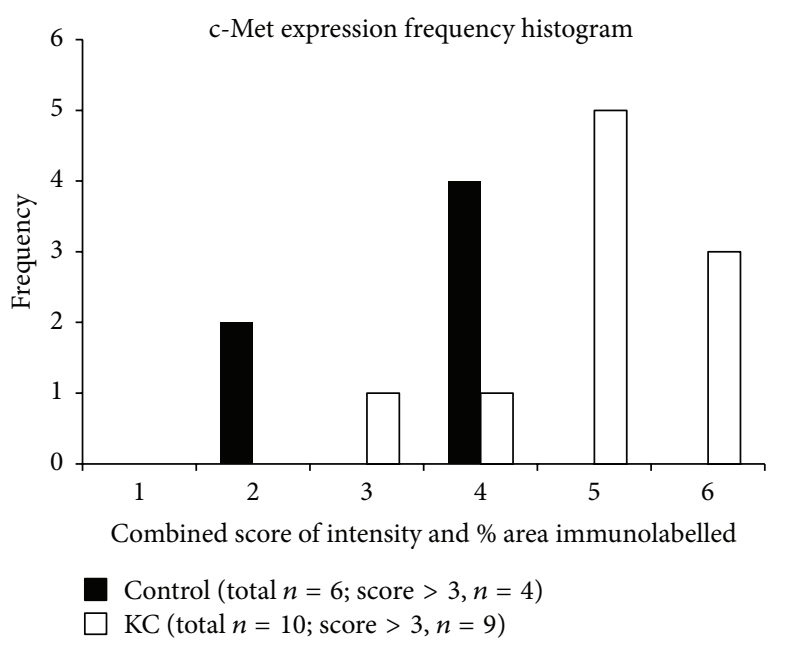

(b)

FIGURE 4: Frequency histograms for HGF (a) and c-Met (b) expression in KC and control corneas (combined score of staining intensity and $\%$ area coverage; range $=0$ to 6 ). Note the right skewed distribution of HGF and c-Met immunostaining for KC corneas compared to controls. HGF staining had a combined score $>3$ in $7 / 10(70 \%)$ KC corneas, compared to $1 / 6(16 \%)$ control corneas. c-Met staining with a combined score $>3$ was seen in $9 / 10$ (90\%) KC corneas compared to $4 / 6$ (66\%) control corneas.

signalling cascades. HGF is a potent enhancer for corneal epithelial cell proliferation [16] and migration [23] in vivo, and increased HGF and c-Met mRNA expressions have been detected during corneal wound healing [16]. Increased HGF protein expression has been detected in the whole corneal epithelium, keratocytes, and tears of wounded compared to unwounded rabbit corneas [21]. HGF mRNA expression was also upregulated in the lacrimal gland when studying injured compared to unwounded rabbit corneas [17]. No difference in c-Met immunostaining was detected between wounded and unwounded rabbit corneas [21]. In secondary injured $\mathrm{KC}$ and control corneas, HGF expression in the stromal cells was reduced [24]. This was suggested to be most likely due to normal tissue repair pathways already being damaged or compromised, implying involvement of HGF in tissue regeneration [24]. These studies also suggested that lacrimal gland and keratocyte-derived HGF are closely associated with corneal tissue repair $[17,24]$. In KC corneal epithelium, we observed increased HGF in the region adjacent to the cone, as well as increased c-Met; however it remains to be determined whether the increased expressions of HGF and c-Met observed are directly involved in KC pathogenesis or are secondary responses.

Poorly regulated and overexpressed HGF may be detrimental to tissues, related to the involvement of HGF in inflammation. For example, delayed formation of the epithelium layer and increased formation of stromal myofibroblasts have been detected during rabbit corneal wound healing, for corneas treated with recombinant HGF [18]. Applying recombinant HGF to corneas of mice with Pseudomonas aeruginosa keratitis also worsened the disease progression, with a significantly higher grade of corneal opacity and thinning compared to PBS-treated corneas [25]. In vitro treatment of corneal stromal keratocytes with proinflammatory interleukin- (IL-) $1 \beta$ increased HGF mRNA and protein production [26]. Elevated corneal HGF expression also enhanced proinflammatory cytokines and decreased anti-inflammatory cytokines in Pseudomonas aeruginosa keratitis in mice, most likely under the control of inflammatory cytokines and cell growth kinases [25].

$\mathrm{KC}$ has traditionally been described as a noninflammatory degenerative condition. However, emerging evidence clearly indicates that inflammation-related processes within the epithelium and stroma are involved in the pathogenesis of KC. Significant increases in proinflammatory molecules such as IL-6, IL-4, IL-5, IL-8, and IL-12 [27-29], MMP-1, MMP-3, MMP-7, and MMP-13 [28], and chemokine C-C motif ligand 5 (CCL5) [29] and significantly decreased levels of lactoferrin, serum albumin, and secreted IgA ( $I I$ A) have been reported in $\mathrm{KC}$ compared to control tears [30]. Keratocytes in KC are reported to express more IL- $1 \alpha$ receptors than controls [31], and one group has proposed that keratocyte apoptosis observed in KC may be induced by the binding of IL-1 receptors secondary to increased levels of IL-1 associated with epithelial trauma, for example, due to eye-rubbing [32]. Most recently, increased mRNA expression of MMP-9 and its inducer proteins IL- 6 and TNF- $\alpha$ were detected in KC corneal epithelium in an Indian cohort (>100 patients) compared to healthy controls $(n=20)$, and tear protein levels of MMP9 and IL-6 were also increased in KC [33]. Treatment with topical cyclosporine A (an immunosuppressant) in a small group of KC patients $(n=20)$ reduced the tear levels of MMP9 and appeared to halt the progression of $\mathrm{KC}$, consistent with the involvement of inflammation in KC pathogenesis [33].

The link between $H G F$ variants and KC susceptibility was first reported by Burdon et al., (2011) and then confirmed in an independent European cohort which replicated the association of SNP rs3735520 and detected a new HGF SNP 
rs2956540 [34]. In addition, an independent study of an Australian population of European descent $(n=830)$ focused on the HGF locus and detected a different SNP (rs4954218) significantly associated with KC [35]. The actual function of these HGF SNPs is unclear. However, a study of rs 3735520 implicated a possible regulatory effect of this SNP for HGF protein expression in serum from KC patients [13]. As the SNPs identified to date are all located in the noncoding region of the gene (rs3735520, rs17501108, and rs1014091 in the upstream of $H G F$ and rs2286194 in the intron between exon 8 and exon 9), it is likely that they will affect gene expression through mechanisms such as RNA splicing, transcription factor binding, and miRNA regulation [36]. Our results provide evidence of increased HGF protein in KC epithelium compared to control corneal epithelium; however the role of the reported HGF SNPs in the increased protein expression is unclear and will be further investigated.

\section{Conclusion}

Previous studies showed an independent, repeatable association between certain SNPs and HGF in KC [13, 34, 35]. The $\mathrm{SNP}$ variations detected were located in the noncoding region of $H G F$ consistent with a role for these SNPs in the regulating HGF expression, and increased serum HGF expression associated with the minor SNP allele has been reported [37]. In the current study, we showed increased HGF protein expression within $\mathrm{KC}$ corneal epithelium. Taken together previous SNP studies, these observations indicate that the HGF/c-Met pathway may be involved in the pathogenesis of KC. Further studies investigating how the HGF/c-Met pathway may be altered in KC, including the associations between SNPs and protein expression and the role of inflammation requires further investigation. Studies on the downstream signalling pathways regulated by HGF/c-Met, such as MAPK cascades, the PI3K-Akt axis, and the JAK/STAT pathway may help to identify the potential role of this pathway in $\mathrm{KC}$ and in normal corneal homeostasis.

\section{Conflict of Interests}

The authors declare that they have no conflict of interests.

\section{Acknowledgments}

The authors thank the Medical School of Sydney University, Sydney Eye Hospital Foundation, and the Lions NSW Eye Bank for funding support. Michele C. Madigan is funded by the National Foundation for Medical Research \& Innovation (NFMRI).

\section{References}

[1] Y. S. Rabinowitz, "Keratoconus," Survey of Ophthalmology, vol. 42, no. 4, pp. 297-319, 1998.

[2] C. N. J. Mcghee, “2008 Sir Norman McAlister gregg lecture: 150 years of practical observations on the conical cornea-what have we learned?" Clinical and Experimental Ophthalmology, vol. 37, no. 2, pp. 160-176, 2009.
[3] H. Wagner, J. T. Barr, and K. Zadnik, "Collaborative Longitudinal Evaluation of Keratoconus (CLEK) Study: methods and findings to date," Contact Lens and Anterior Eye, vol. 30, no. 4, pp. 223-232, 2007.

[4] K. A. Williams, M. T. Lowe, M. C. Keane et al., The Australian Corneal Graft Registry 2012 Report, Flinders University Press, Adelaide, Australia, 2012.

[5] G. R. Snibson, "Collagen cross-linking: a new treatment paradigm in corneal disease-a review," Clinical and Experimental Ophthalmology, vol. 38, no. 2, pp. 141-153, 2010.

[6] K. van Dijk, V. S. Liarakos, J. Parker et al., "Bowman layer transplantation to reduce and stabilize progressive, advanced keratoconus," Ophthalmology, vol. 122, no. 5, pp. 909-917, 2015.

[7] E. Sykakis, F. Carley, L. Irion, J. Denton, and M. C. Hillarby, "An in depth analysis of histopathological characteristics found in keratoconus," Pathology, vol. 44, no. 3, pp. 234-239, 2012.

[8] J. C. Fan Gaskin, I. P. Loh, C. N. McGhee, and T. Sherwin, "An immunohistochemical study of inflammatory cell changes and matrix remodeling with and without acute hydrops in keratoconus," Investigative Opthalmology \& Visual Science, vol. 56, no. 10, pp. 5831-5837, 2015.

[9] L. Zhou, B. Y. J. T. Yue, S. S. Twining, J. Sugar, and R. S. Feder, "Expression of wound healing and stress-related proteins in keratoconus corneas," Current Eye Research, vol. 15, no. 11, pp. 1124-1131, 1996.

[10] I. Lema and J. A. Durán, "Inflammatory molecules in the tears of patients with keratoconus," Ophthalmology, vol. 112, no. 4, pp. 654-659, 2005.

[11] V. Galvis, T. Sherwin, A. Tello, J. Merayo, R. Barrera, and A. Acera, "Keratoconus: an inflammatory disorder?" Eye, vol. 29, no. 7, pp. 843-859, 2015.

[12] Y.-T. Huang, C.-J. Chang, and K.-M. Chao, "The extent of linkage disequilibrium and computational challenges of single nucleotide polymorphisms in genome-wide association studies," Current Drug Metabolism, vol. 12, no. 5, pp. 498-506, 2011.

[13] K. P. Burdon, S. Macgregor, Y. Bykhovskaya et al., "Association of polymorphisms in the hepatocyte growth factor gene promoter with keratoconus," Investigative Ophthalmology \& Visual Science, vol. 52, no. 11, pp. 8514-8519, 2011.

[14] S. L. Organ and M. Tsao, "An overview of the c-MET signaling pathway," Therapeutic Advances in Medical Oncology, vol. 3, no. 1, supplement, pp. S7-S19, 2011.

[15] J. T. Daniels, G. A. Limb, U. Saarialho-Kere, G. Murphy, and P. T. Khaw, "Human corneal epithelial cells require MMP-1 for HGFmediated migration on collagen I," Investigative Ophthalmology and Visual Science, vol. 44, no. 3, pp. 1048-1055, 2003.

[16] S. E. Wilson, J. W. Walker, E. L. Chwang, and Y.-G. He, "Hepatocyte growth factor, keratinocyte growth factor, their receptors, fibroblast growth factor receptor-2, and the cells of the cornea," Investigative Ophthalmology and Visual Science, vol. 34, no. 8, pp. 2544-2561, 1993.

[17] S. E. Wilson, Q. Liang, and W. J. Kim, "Lacrimal gland HGF, KGF, and EGF mRNA levels increase after corneal epithelial wounding," Investigative Ophthalmology and Visual Science, vol. 40, no. 10, pp. 2185-2190, 1999.

[18] L. M. Carrington and M. Boulton, "Hepatocyte growth factor and keratinocyte growth factor regulation of epithelial and stromal corneal wound healing," Journal of Cataract and Refractive Surgery, vol. 31, no. 2, pp. 412-423, 2005.

[19] J. You, L. Wen, A. Roufas, M. C. Madigan, and G. Sutton, "Expression of SFRP family proteins in human keratoconus corneas," PLoS ONE, vol. 8, no. 6, Article ID e66770, 2013. 
[20] D.-Q. Li and S. C. G. Tseng, “Three patterns of cytokine expression potentially involved in epithelial-fibroblast interactions of human ocular surface," Journal of Cellular Physiology, vol. 163, no. 1, pp. 61-79, 1995.

[21] Q. Li, J. Weng, R. R. Mohan et al., "Hepatocyte growth factor and hepatocyte growth factor receptor in the lacrimal gland, tears, and cornea," Investigative Ophthalmology and Visual Science, vol. 37, no. 5, pp. 727-739, 1996.

[22] S. E. Wilson, Y.-G. He, J. Weng, J. D. Zieske, J. V. Jester, and G. S. Schultz, "Effect of epidermal growth factor, hepatocyte growth factor, and keratinocyte growth factor, on proliferation, motility and differentiation of human corneal epithelial cells," Experimental Eye Research, vol. 59, no. 6, pp. 665-678, 1994.

[23] J. K. Spix, E. Y. Chay, E. R. Block, and J. K. Klarlund, "Hepatocyte growth factor induces epithelial cell motility through transactivation of the epidermal growth factor receptor," Experimental Cell Research, vol. 313, no. 15, pp. 3319-3325, 2007.

[24] I. M. Cheung, C. N. McGhee, and T. Sherwin, "Deficient repair regulatory response to injury in keratoconic stromal cells," Clinical and Experimental Optometry, vol. 97, no. 3, pp. 234-239, 2014.

[25] X. Jiang, S. A. McClellan, R. Barrett, M. Foldenauer, and L. D. Hazlett, "HGF signaling impacts severity of Pseudomonas aeruginosa keratitis," Investigative Ophthalmology and Visual Science, vol. 55, no. 4, pp. 2180-2190, 2014.

[26] J. Weng, R. R. Mohan, Q. Li, and S. E. Wilson, "IL-1 upregulates keratinocyte growth factor and hepatocyte growth factor mRNA and protein production by cultured stromal fibroblast cells: interleukin-1 $\beta$ expression in the cornea," Cornea, vol. 16, no. 4, pp. 465-471, 1997.

[27] I. Lema, T. Sobrino, J. A. Durán, D. Brea, and E. Díez-Feijoo, "Subclinical keratoconus and inflammatory molecules from tears," British Journal of Ophthalmology, vol. 93, no. 6, pp. 820824, 2009.

[28] S. A. Balasubramanian, S. Mohan, D. C. Pye, and M. D. P. Willcox, "Proteases, proteolysis and inflammatory molecules in the tears of people with keratoconus," Acta Ophthalmologica, vol. 90, no. 4, pp. e303-e309, 2012.

[29] A. S. Jun, L. Cope, C. Speck et al., "Subnormal cytokine profile in the tear fluid of keratoconus patients," PLOS ONE, vol. 6, Article ID e16437, 2011.

[30] S. A. Balasubramanian, D. C. Pye, and M. D. P. Willcox, "Levels of lactoferrin, secretory IgA and serum albumin in the tear film of people with keratoconus," Experimental Eye Research, vol. 96, no. 1, pp. 132-137, 2012.

[31] E. J. Fabre, J. Bureau, Y. Pouliquen, and G. Lorans, "Binding sites for human interleukin $1 \alpha$ gamma interferon and tumor necrosis factor on cultured fibroblasts of normal cornea and keratoconus," Current Eye Research, vol. 10, no. 7, pp. 585-592, 1991.

[32] N. Efron and J. G. Hollingsworth, "New perspectives on keratoconus as revealed by corneal confocal microscopy," Clinical and Experimental Optometry, vol. 91, no. 1, pp. 34-55, 2008.

[33] R. Shetty, A. Ghosh, R. R. Lim et al., "Elevated expression of matrix metalloproteinase- 9 and inflammatory cytokines in keratoconus patients is inhibited by cyclosporine A," Investigative Ophthalmology and Visual Science, vol. 56, no. 2, pp. 738-750, 2015.

[34] L. Dudakova, M. Palos, K. Jirsova et al., "Validation of rs2956540:G>C and rs3735520:G>A association with keratoconus in a population of European descent," European Journal of Human Genetics, vol. 23, no. 11, pp. 1581-1583, 2015.
[35] S. Sahebjada, M. Schache, A. J. Richardson, G. Snibson, M. Daniell, and P. N. Baird, "Association of the hepatocyte growth factor gene with Keratoconus in an Australian population," PLoS ONE, vol. 9, no. 1, Article ID e84067, 2014.

[36] Q. Huang, "Genetic study of complex diseases in the postGWAS era," Journal of Genetics and Genomics, vol. 42, no. 3, pp. 87-98, 2015.

[37] K. P. Burdon, S. Macgregor, Y. Bykhovskaya et al., "Association of polymorphisms in the hepatocyte growth factor gene promoter with keratoconus," Investigative Ophthalmology and Visual Science, vol. 52, no. 11, pp. 8514-8519, 2011. 


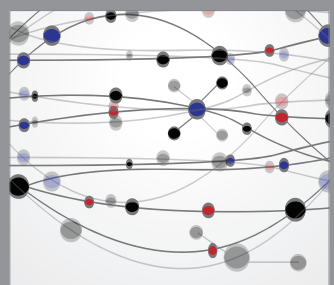

The Scientific World Journal
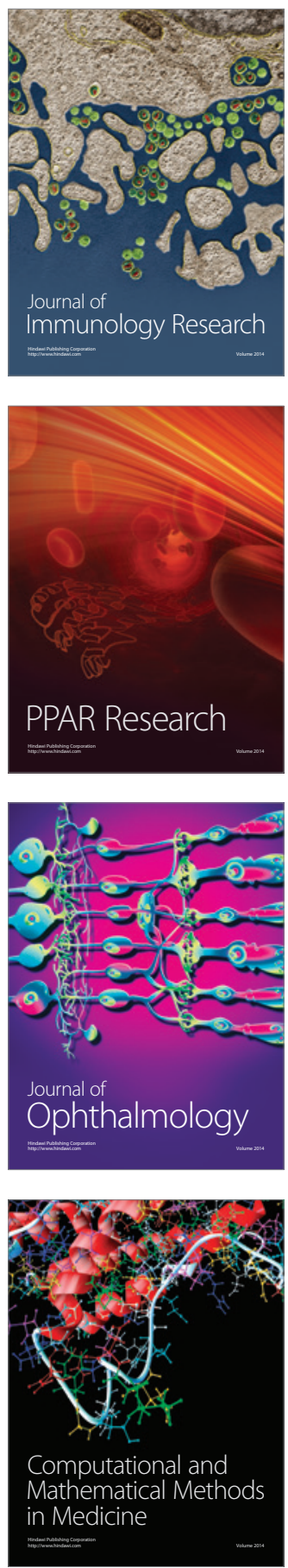

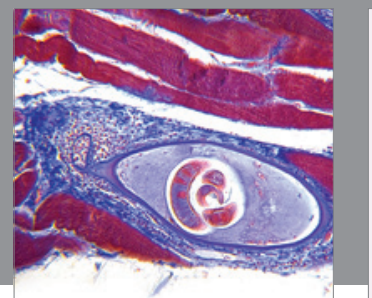

Gastroenterology

Research and Practice
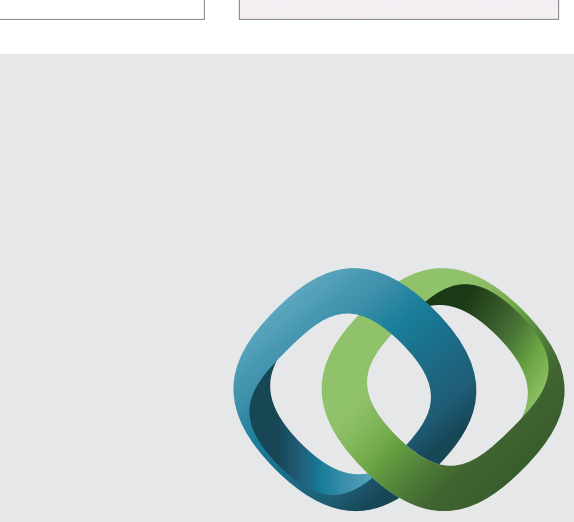

\section{Hindawi}

Submit your manuscripts at

http://www.hindawi.com
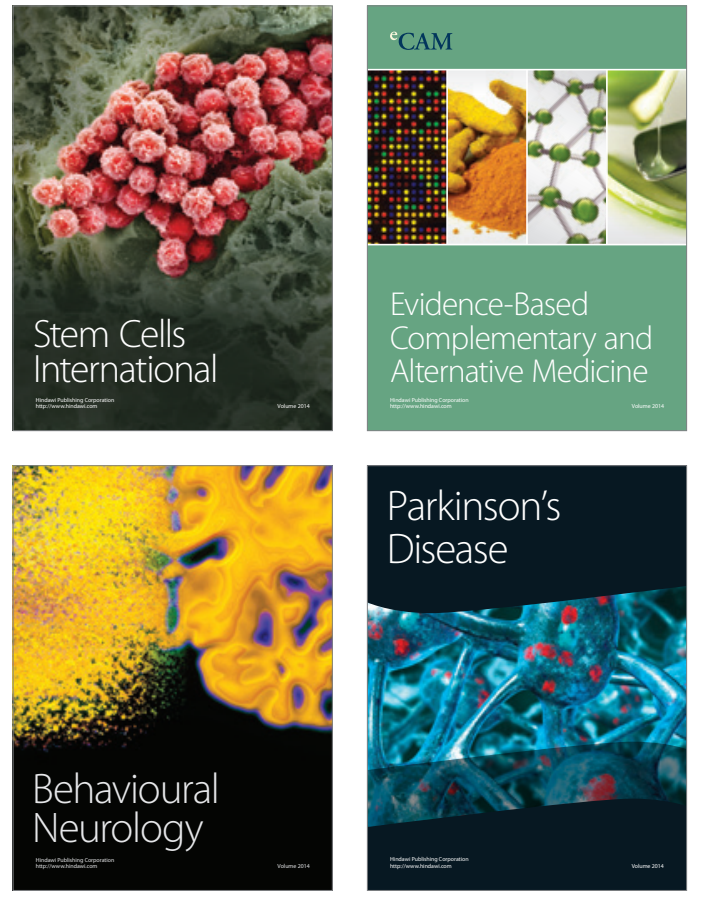
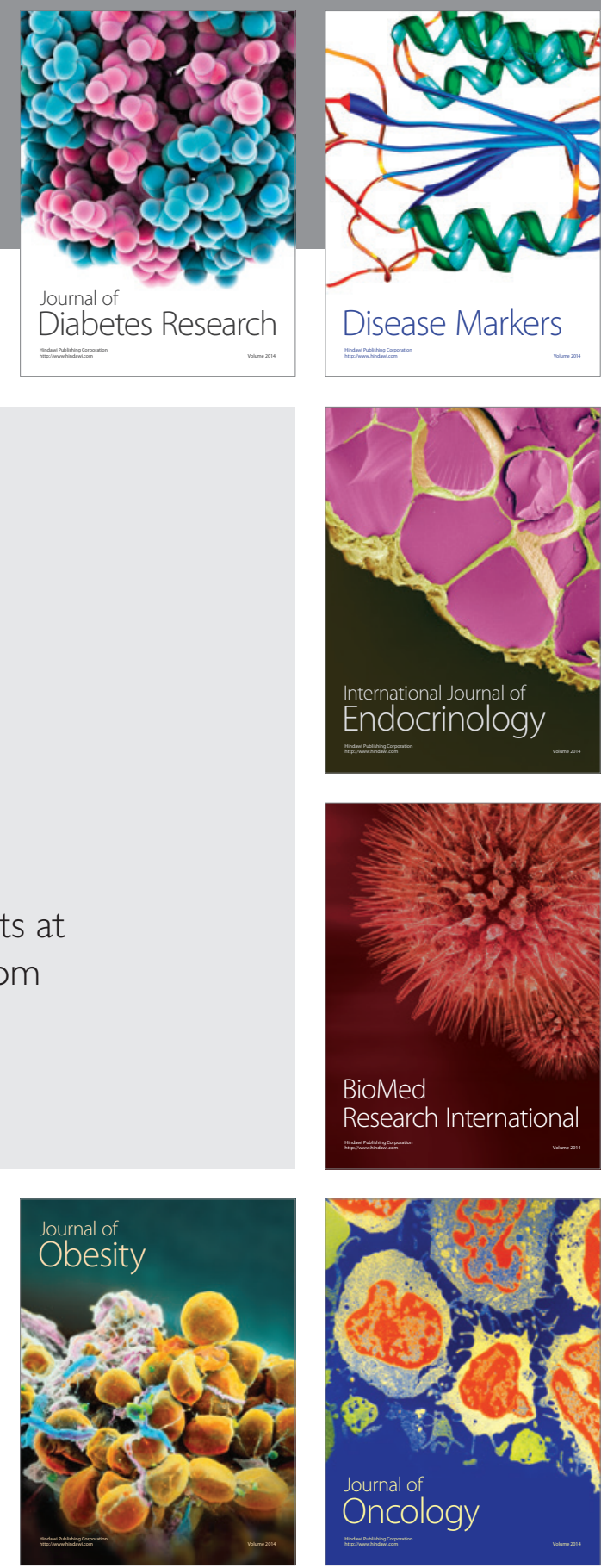

Disease Markers
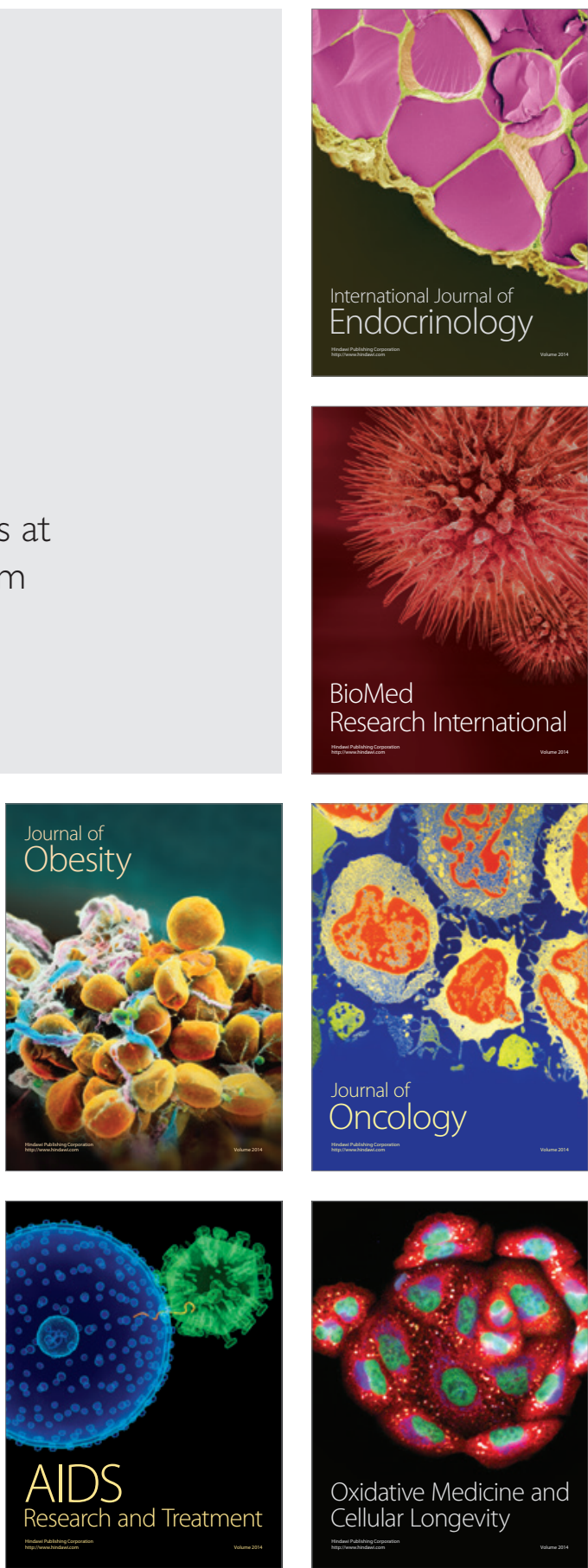